\title{
LA VISITA A PRISIÓN COMO METODOLOGÍA INNOVADORA EN DERECHO PENAL
}

\author{
Deborah García-Magna1: Universidad de Málaga. España
}

dgmagna@uma.es

José Becerra-Muñoz: Universidad de Málaga. España

josebecerra@uma.es

\section{RESUMEN}

Cada curso académico, como actividad complementaria a la metodología docente en el Área de Derecho Penal de la Universidad de Málaga, se realiza desde hace ya algunos años una visita guiada a la prisión para que los alumnos conozcan de primera mano la realidad de la sanción penal que con más frecuencia se aplica en nuestro ordenamiento jurídico. En este artículo describimos cómo se organiza la actividad, qué objetivos concretos tiene y qué grado de éxito estamos obteniendo. Sucintamente, pretendemos que los estudiantes adquieran conciencia crítica sobre la naturaleza represiva del Derecho Penal y sus diversos instrumentos de control, además de desarrollar la capacidad para valorar la realidad normativa penal y para detectar sus fines y efectos secundarios no explícitos. Para poder verificar si se cumplen dichos objetivos hemos llevado a cabo un estudio empírico mediante encuestas de valoración que los estudiantes han completado después de realizar la visita. Destacamos que un porcentaje muy elevado de los alumnos considera que la actividad es una experiencia imprescindible para su formación como juristas y también para el desarrollo personal como individuos con capacidad crítica y conciencia social.

PAlABRAS CLAVE: Actividades - Visitas- Competencias - Analogía personal Valoración 


\title{
VISITING PRISONS AS AN INNOVATIVE TEACHING METHODOLOGY IN CRIMINAL LAW
}

\begin{abstract}
Every year, as a complementary activity to the teaching methodology in the Criminal Law Department, guided visits to prison facilities are organized so students can meet face to face the reality of this criminal sanction, the most frequently applied penalty of our criminal legal system. In this article we describe how this activity is organized, which are the specific aims of it and to what extent we are successfully pursuing them. Briefly, our main objective is for students to acquire a critical approach about the repressive nature of Criminal Law and its various instruments of control, in addition to the enhancement of their capacity to analyze the criminal legal reality and to detect its secondary non-explicit goals and effects. To verify the accomplishment of such objectives we have carried out an empirical study throughout evaluation surveys filled in by students after their participation in the activity. We emphasize a very high percentage of students that consider the visit an essential activity for their education as jurists but also as critical and socially committed individuals.
\end{abstract}

KEY WORDS: Activities - Visits- Abilities - Personal analogy - Evaluation

\section{INTRODUCCIÓN}

\subsection{La docencia del Derecho Penal en el contexto del EEES}

Las Universidades están viviendo momentos de profundo cambio que necesariamente afectarán a todas las personas implicadas, empezando por los propios estudiantes. Quienes estamos comprometidos con la docencia sabemos bien que, como afirma Bain (2005, pág. 28), "el aprendizaje tiene poco sentido si no es capaz de producir una influencia duradera e importante en la manera en que la gente actúa, piensa y siente".

La demanda de una Universidad basada en un enfoque abierto de la docencia (Terradillos Basoco, 2008, p. 6) que ponga el acento en la calidad del conocimiento adquirido (González Rus, 2003, p. 12) no es nueva. Se precisa un cambio cultural global que supondrá para los alumnos la adaptación a un nuevo modelo metodológico de aprendizaje activo (Bricall, 2000); (Gil Flores et al., 2004); (Esteve, 
El proceso en el que nos encontramos inmersos corre, sin embargo, el riesgo de arrastrarnos hacia un sistema excesivamente dirigido a la empleabilidad, que puede hacer entrar en crisis la idea misma de formación que se pretende con la reforma y dejar en un segundo plano valores y principios que tradicionalmente han caracterizado al estudiante universitario, tales como, "la contestación, la crítica, la solidaridad, la reflexión sosegada, la vida cultural", etc. (Zabalza, 2009, p. 26-28), tan necesarios en el desarrollo de la personalidad, también misión fundamental de la Universidad. En definitiva, la reforma de la enseñanza y del pensamiento han de ir de la mano (Morin, 2001, p. 23).

Estamos convencidos de que el estudiante de Derecho Penal no puede limitarse a asimilar los contenidos de la materia, sino que debe acercarse con actitud crítica a la realidad de su aplicación sobre individuos concretos. Así, se hace preciso proporcionar al alumno un conocimiento que vaya más allá del análisis de las normas como conceptos teóricos, preparándole para que comprenda también sus implicaciones fácticas. A través de las metodologías propias de las ciencias sociales se debe analizar el delito, los factores que lo desencadenan, la realidad de su gravedad y frecuencia, las características de los autores y las víctimas, y los durísimos efectos psicosociales que la prisión tiene sobre los individuos sometidos a privación de libertad $^{2}$. En definitiva, los profesores debemos encontrar la forma de que el alumno se plantee por sí mismo las grandes preguntas sobre el hecho social que es el delito (Terradillos Basoco, 2008, p. 8) y que tenga la actitud crítica suficiente para buscar las respuestas.

Tradicionalmente las universidades europeas han dado prioridad a los contenidos teóricos, presuponiendo que con su conocimiento se adquirían las habilidades necesarias en cada titulación (García Magna et al., 2011, p. 388). El nuevo modelo educativo incorpora la necesidad de trabajar también la interrelación con los demás (comprendiendo y aceptando la diversidad) y el desarrollo de la personalidad, de la autonomía personal y de la responsabilidad (Delors, 1996).

En ese sentido, nos interesa que nuestros estudiantes adquieran conciencia crítica sobre la naturaleza represiva del Derecho Penal y sus diversos instrumentos de control (competencia específica de carácter cognitivo) y que desarrollen la capacidad para valorar la realidad normativa penal y para detectar sus fines y efectos secundarios no explícitos (competencia específica de carácter actitudinal). 
El reto está en diseñar una programación docente que nos permita hacer realidad esa decisión metodológica ${ }^{3}$.

\subsection{La visita a prisión como metodología innovadora}

Las estrategias docentes que mejor consiguen que el estudiante desarrolle competencias de tipo actitudinal son aquellas basadas en la analogía personal, es decir, actividades que buscan que "el individuo se identifique personalmente con el problema para verlo desde dentro" (Jiménez Martín \& Moncholi Chaparro, 2009. p. 8).

En las visitas a prisión el estudiante se ve confrontado con una realidad que apenas conoce y que le puede ayudar a conseguir una mínima comprensión de lo que supone vivir encerrado en un establecimiento penitenciario, con la obvia repercusión que ello tiene sobre el individuo a nivel psicológico, emocional y social. Con esta actividad, por tanto, el estudiante puede experimentar empatía con la persona que se encuentra privada de libertad (o con aquellas otras que trabajan en dichos establecimientos: juristas, psicólogos, trabajadores sociales, personal de seguridad, etc.).

Las visitas se suelen realizar al final del curso, como una manera de confrontar lo aprendido en el aula durante el año con lo que ocurre en la realidad cotidiana del ejercicio de profesiones relacionadas con sus estudios. A los alumnos se les proporciona previamente la información esencial para que acudan a la visita sabiendo qué van a ver ${ }^{4}$.

A nivel organizativo, la Dirección General de Instituciones Penitenciarias debe conceder la autorización pertinente y la propia Dirección del Centro establece el número de estudiantes que podrá asistir cada día (entre 20 y 25), las fechas concretas y los módulos y estancias que se podrán visitar. Contamos con la inestimable colaboración de los juristas del centro, que acompañan a los estudiantes durante el recorrido, resolviendo las dudas que surgen.

\footnotetext{
${ }^{3}$ TORRANCE, E.P. y MYERS, R.E. (1976), La enseñanza creativa, Madrid, Santillana, recomiendan que el maestro sea creativo, para conseguir que los alumnos también lo sean. FERNÁNDEZ MARCH (2006), quienes destacan que en dicha programación docente deben estar presentes tanto los objetivos formativos como las actividades que deberán realizar los alumnos, transmitiéndoles de manera clara
} 
Normalmente la actividad incluye la visita a varios módulos, talleres y locutorios de comunicación con abogados. Los estudiantes tienen libertad para hablar con quien deseen (reclusos y/o personal del centro). Además, se suele preparar un debate para después de la visita, que se realiza en el mismo centro, con la participación de algunos internos.

\subsection{Evaluando la visita a prisión}

Una cuestión esencial para asegurar que la actividad está teniendo los efectos pretendidos es obtener la imprescindible retroalimentación por parte de los alumnos. Todos los docentes que participan en la actividad tienen la impresión, por comentarios de los propios alumnos durante y después de la experiencia, de que la valoración que realizan de la actividad es sumamente positiva, pero se hace necesaria una evaluación sistemática, científicamente válida y que permita indagar en los múltiples aspectos que componen la actividad de manera ordenada.

\section{METODOLOGÍA}

Para ello, hemos elaborado un cuestionario que nos permitirá conocer la opinión de los alumnos que han realizado la visita a prisión. En su confección hemos tomado como referencia la "Encuesta de Opinión del Alumnado sobre la Actuación Docente del Profesorado" elaborada por el Vicerrectorado de Calidad de la Universidad de Málaga, así como un cuestionario genérico sobre satisfacción con actividades varias confeccionado por el Decanato de la Facultad de Derecho en el marco de su Sistema de Garantía de la Calidad (SGC) ${ }^{5}$.

A pesar de ello, el cuestionario finalmente elaborado es en gran medida original debido a las especiales características de la actividad que pretende evaluar, así como al proceso de concreción de las preguntas que realizamos para evitar un cuestionario demasiado largo y asegurar el mayor número de respuestas posibles ${ }^{6}$.

El resultado es un instrumento que se compone de nueve preguntas de respuesta múltiple (cerradas) en alguna de las cuales se pide al entrevistado que elija entre las respuestas ofrecidas y en otras que valore su grado de acuerdo con el enunciado planteado (nada, poco, bastante o mucho). Estas se complementan además con la posibilidad en diversos puntos de la encuesta de realizar comentarios libres (respuestas abiertas). Esta alternativa enriquece considerablemente el cuestionario permitiendo pulsar con mucha más precisión el sentir del encuestado y proporcionándole una vía para incluir cuestiones que, a su juicio, no quedan 
debidamente recogidas en el cuestionario o en las que no siente haber podido expresarse correctamente por lo cerrado de las respuestas.

Para su elaboración y distribución se utilizó la "Encuesta personalizable" de la herramienta de teleformación Campus Virtual, un entorno virtual de enseñanzaaprendizaje desarrollado a partir de Moodle, por el Servicio de Enseñanza Virtual de la Universidad de Málaga7. A nuestro juicio, se trata de la herramienta idónea para ello debido a que en dicho entorno virtual se encuentran todos los alumnos matriculados en la asignatura "Derecho Penal. Parte general" de la Licenciatura en Derecho, que son los destinatarios de la actividad a evaluar.

Durante el proceso de elaboración de la misma, la encuesta se sometió, además, a la valoración de todos los integrantes del Área de Derecho Penal, que pudieron proponer modificaciones, adiciones o supresiones a la misma.

Una vez terminada, la encuesta se puso a disposición de los alumnos en la plataforma virtual para su realización durante un mes (junio-julio), remitiéndoseles un correo electrónico explicando el contenido y los objetivos de la encuesta, así como asegurándoseles su total anonimato y desvinculación con la evaluación de la asignatura.

Se obtuvieron un total de 55 respuestas de un universo de 246 sujetos $^{8}$, por lo que la tasa de respuesta es baja, como es habitual cuando las encuestas se realizan por correo (Varona Gómez, 2008. Pág. 5; Roldán Barbero, 2009. Pág. 71), circunstancia que aumenta sensiblemente el margen de error admitido en los resultados.

Junto a ello, debido a que no se ha utilizado un método probabilístico para seleccionar la muestra, debemos ser muy cautos a la hora de extrapolar estos datos al resto de la población. Se trata, sin embargo, de un sesgo típico de los estudios exploratorios o pilotos, que esperamos ir descartando progresivamente a medida que la investigación avance. Los datos de la muestra son:
a) $\mathrm{N}$ (población total) $=246$
b) $\mathrm{K}$ [error de muestreo para nivel de confianza $(\mathrm{z})=95 \%]=2$
c) e (margen de error) $=0,11$
d) $P=Q($ varianza poblacional $)=50 \%$
e) $\mathrm{n}$ (tamaño de la muestra) $=55$ 


\section{ANÁLISIS Y DISCUSIÓN}

Por lo que se refiere a las respuestas obtenidas por parte de los estudiantes constatamos que, en primer lugar, la inmensa mayoría de los encuestados (96\%) decidieron participar en la visita desde que se anunció a principios de curso, tan sólo el $4 \%$ restante no estaba seguro al principio pero se fue decidiendo a ello a medida que avanzaba el curso. De ello podemos deducir que se trata de una actividad que motiva considerablemente a los estudiantes y que no despierta recelos o temores por su aparente peligrosidad.

La mayoría de ellos (83\%) está de acuerdo en que el mejor momento para llevar a cabo la visita es al final del curso, una vez que ya se han estudiado las bases del sistema penal, cuando efectivamente se lleva a cabo.

En cuanto al conocimiento que creen tener de la institución penitenciaria, dicen saber poco de ella antes de la visita y bastante una vez que la han realizado, de lo que podemos deducir que se encuentran satisfechos de la información y vivencia que la experiencia les transmite.

Una pregunta de gran interés para conocer su impresión de la visita a prisión es la que pretende averiguar en qué medida les ha sorprendido la actividad. Este aspecto del cuestionario va más allá de las preguntas anteriores, en las que se constata que efectivamente consideran que han aprendido, y permite confirmar que la mayoría de los alumnos tienen instaladas una serie de concepciones erróneas acerca de la institución penitenciaria que no se ven confirmadas mediante la visita. El alumno, por tanto, tiene la impresión de que "descubre" una prisión distinta a la que habría esperado encontrar y cuyas características ha podido obtener de los medios de comunicación o el cine, por ejemplo.

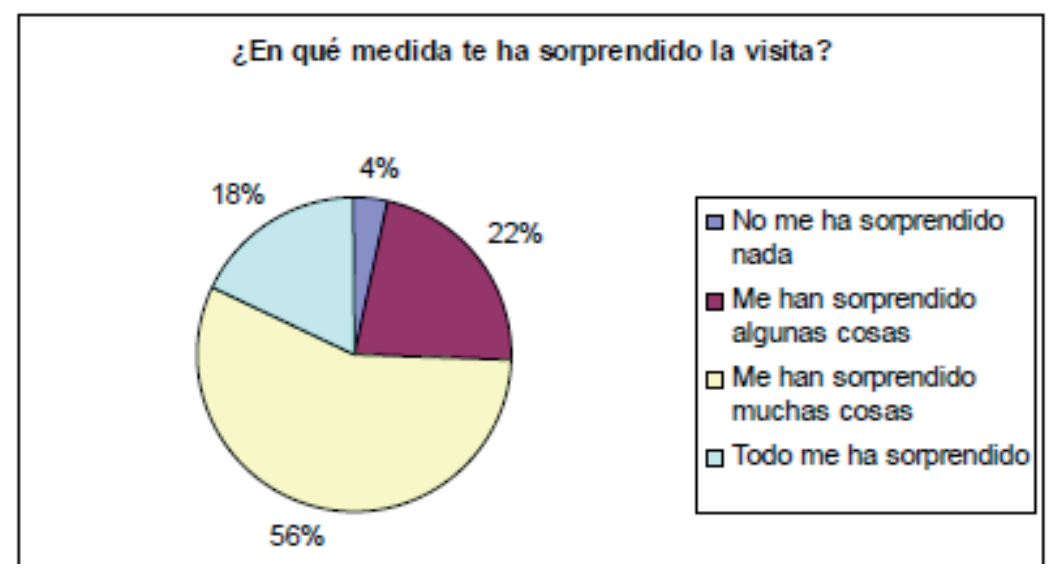


Como se puede ver en el gráfico anterior, Los resultados reflejan que casi tres cuartas partes $(74 \%)$ de los alumnos que han respondido consideran que les han sorprendido muchas cosas o todo en la visita, mientras que tan sólo un $4 \%$ de ellos esperaba lo que ha tenido oportunidad de conocer durante la actividad.

Tal información se complementa, además, con la posibilidad de realizar comentarios al respecto por parte del encuestado, en los que algunos alumnos hacen referencia a lo sorprendente de la visita para ellos, con frases como: "la visita a las diferentes celdas me llamó mucho la atención porque me esperaba otra cosa... [tienen televisores, comparten la celda (lo que debe ser incómodo para hacer sus necesidades, etc)]. En general, me ha gustado mucho la visita a prisión porque tenía una visión totalmente distinta de la misma"; "Pude apreciar como muchas de las cosas que he estudiado no se llevan a cabo"; o "Cuando sales del centro penitenciario tienes la sensación de llevarte conocimientos e impresiones nuevas, más reales, que sin esa visita no podrías haber adquirido".

Una vez obtenida una impresión general de la visita, hemos considerado importante solicitar una valoración más específica de los diferentes aspectos que la componen. Así, se les pide que valoren en una escala que va desde "No me ha gustado" hasta "Me ha parecido imprescindible" diferentes aspectos de la visita.

De los diferentes ítems por los que son preguntados, la actividad que los alumnos consideran más importante es la charla informal que se produce con los internos dentro de los módulos. Generalmente, el grupo de alumnos entra en alguno de ellos donde existe un espacio cubierto con mesas, sillas y una televisión, donde los internos están charlando entre ellos o jugando a algún juego de mesa.

A continuación se accede a un patio al aire libre donde de nuevo los presos charlan, pasean, corren, juegan al fútbol o al baloncesto. Los alumnos, acompañados del jurista y del profesor, pueden entablar en estas dependencias un diálogo con los internos que lo deseen, formándose diferentes grupos y conversaciones en la mayoría de las cuales ninguna de las dos figuras de autoridad participa. De esta manera, tanto alumnos como internos se comunican con total libertad, dependiendo exclusivamente de su capacidad para interactuar mutuamente.

Como decimos, la valoración de esta actividad es sumamente positiva, de manera que, como se observa en el siguiente gráfico, el 73\% de los encuestados la considera imprescindible. Las tres respuestas positivas disponibles en el cuestionario agrupan el $98 \%$ de las respuestas, siendo inexistentes las respuestas negativas o indiferentes. 


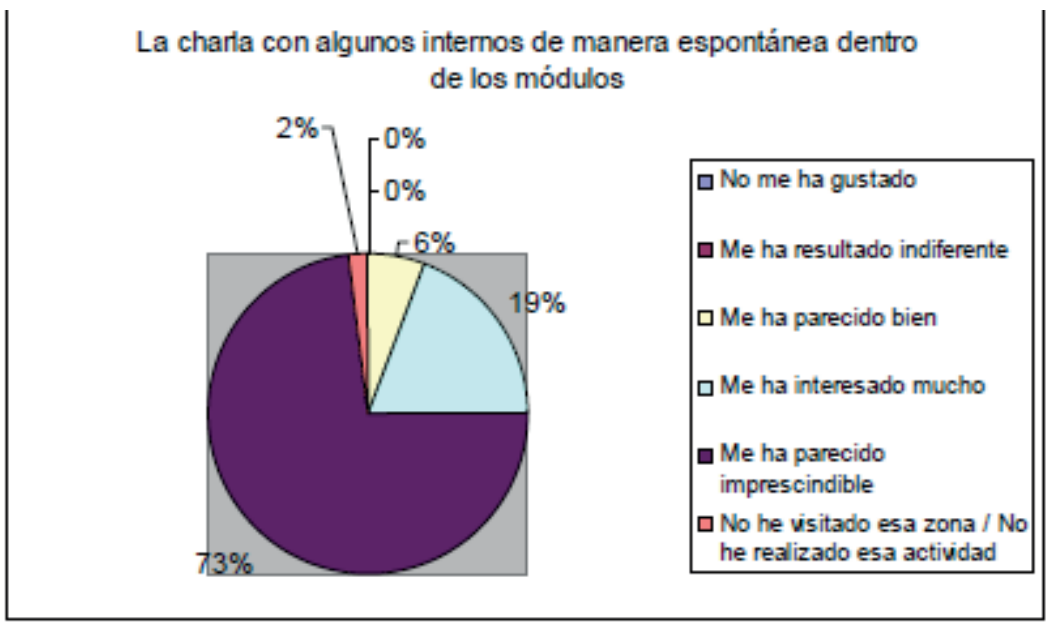

Figura 2. Charla informal con internos.

Fuente: elaboración propia.

Un resultado similar arrojan las respuestas sobre la charla informal con los funcionarios que se encuentran en los mismos módulos. En este caso se produce una situación parecida a la anterior y la percepción de los alumnos es también muy positiva. Las respuestas positivas abarcan el $94 \%$ de todas ellas, si bien algo más repartidas que en el caso anterior. Surge alguna voz disconforme o indiferente respecto a esta parte de la visita, así como un escaso porcentaje (menos que en el caso anterior) que no la ha llevado a cabo.

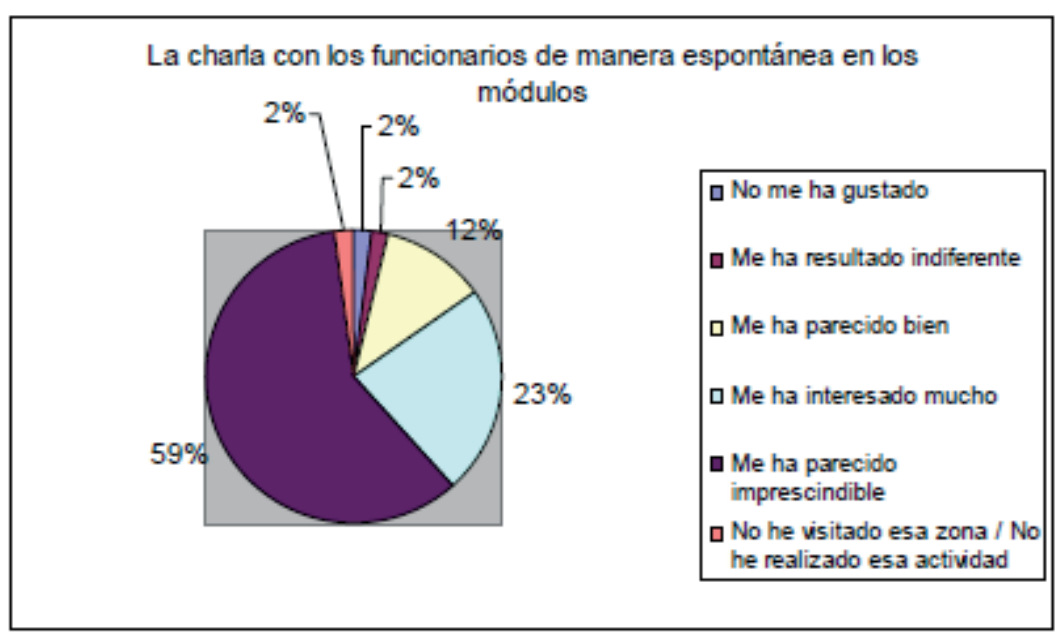

Figura 3. Charla informal con funcionarios.

Fuente: elaboración propia. 


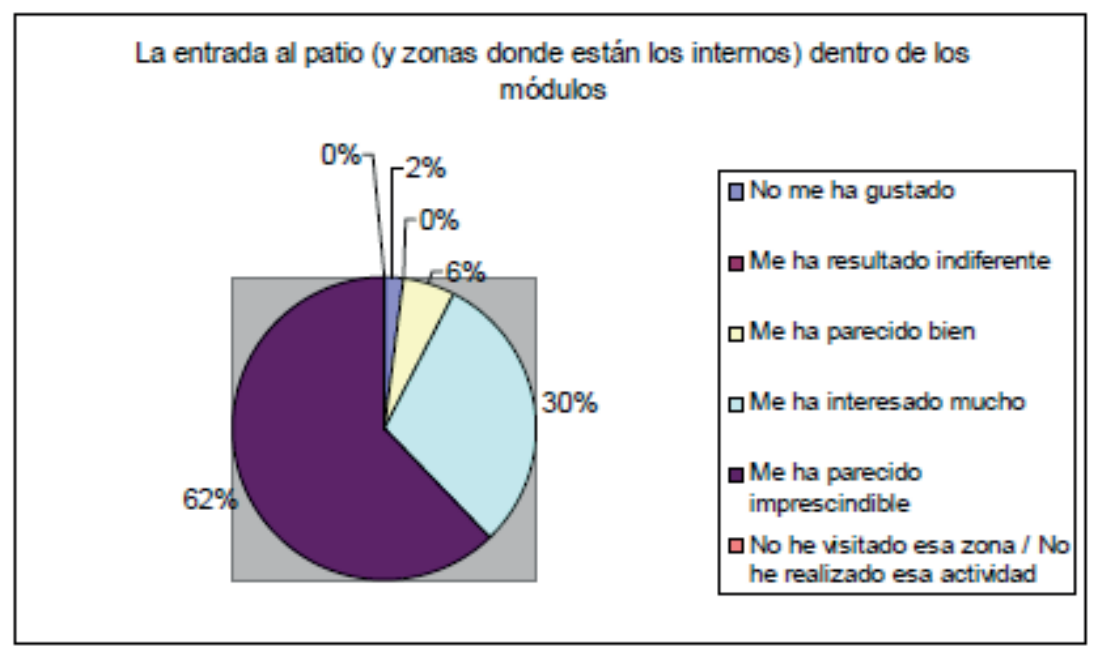

Figura 4. Entrada a módulos.

Fuente: elaboración propia.

Otra actividad que se lleva a cabo en dichos módulos es la visita a la planta superior de los mismos, donde se encuentran las celdas. En este caso los alumnos van acompañados del funcionario o funcionarios del módulo, del jurista y del profesor, mientras que los internos suelen permanecer en las zonas comunes de la planta baja. En este caso, de nuevo, una mayoría considerable de los alumnos valora positivamente la actividad (98\%), siendo el 69\% los que la consideran imprescindible, si bien de nuevo surge alguna voz crítica que declara no haberle gustado la experiencia concreta.

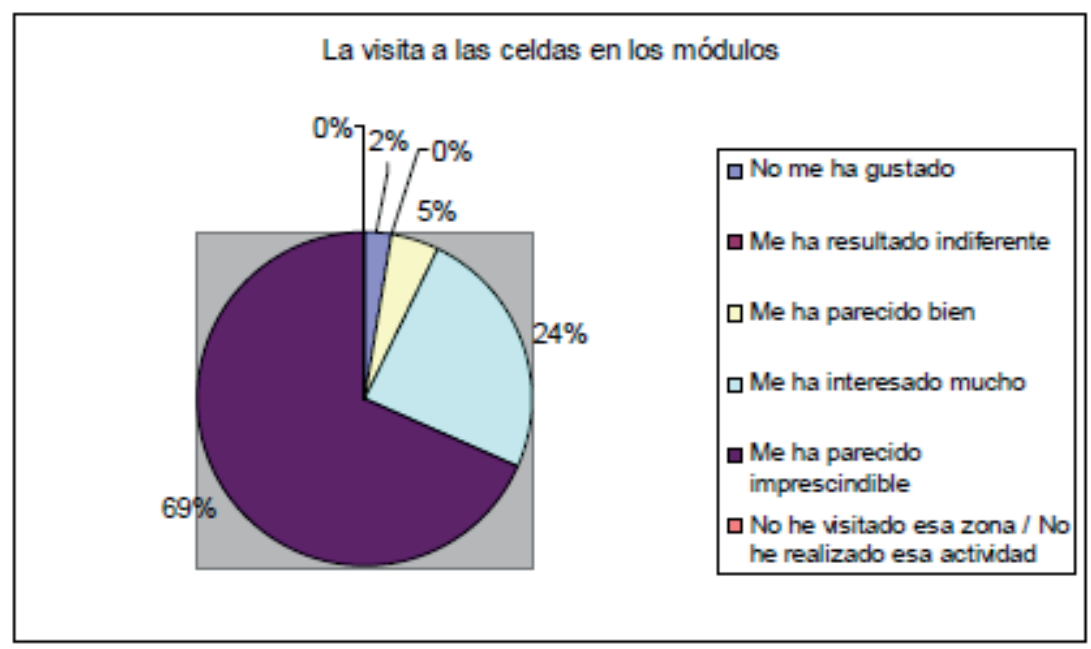

Figura 5. Visita a las celdas. 
Por lo que respecta al recorrido por el resto de las instalaciones, aunque es común la afirmación de que en prisión todo contribuye al tratamiento de los internos, hemos optado por separar la visita a zonas comunes dedicadas a actividades diversas de los internos o a la simple gestión del centro (piscina, polideportivo, salón de actos, cocina, etc.), de la visita a los talleres, en los que se realizan actividades de formación específica para la inserción laboral del interno y para mejorar su capacidad de reinserción en la sociedad.

Los alumnos están generalmente satisfechos con el primer grupo de actividades, si bien comprobamos que se trata de la variable en la que más se ha respondido "No he visitado esa zona/No he realizado esa actividad" (32\%).

En cuanto a la visita a los talleres, la satisfacción es algo menor en comparación con las actividades vistas hasta el momento, las respuestas positivas agrupan el $84 \%$ de ellas, predominando las respuestas "Me ha interesado mucho". Junto a ello, de nuevo las respuestas del tipo "No he visitado esa zona/No he realizado esa actividad" son comunes, pero en mucha menos medida que en el caso anterior (tan sólo el 11\%), y a un $4 \%$ de los encuestados no le ha gustado la actividad o le ha resultado indiferente (véase el siguiente gráfico que muestra los datos absolutos).

Una vez dicho lo anterior, hay dos actividades cuya valoración es claramente discordante respecto al resto: se trata de la visita a los locutorios donde los abogados se entrevistan con los internos y la charla que el jurista da al iniciar la visita.

En la primera de estas dos situaciones resulta muy llamativo que la valoración no sea la más alta. Tanto los profesionales del centro como los profesores que organizan la visita suelen considerar evidente que los estudiantes de Derecho tendrán interés en conocer el lugar donde el abogado, una de las profesiones más comunes del licenciado en Derecho, ejerce su labor en el centro penitenciario. Sin embargo, no parece que el alumno lo perciba de esa manera: 


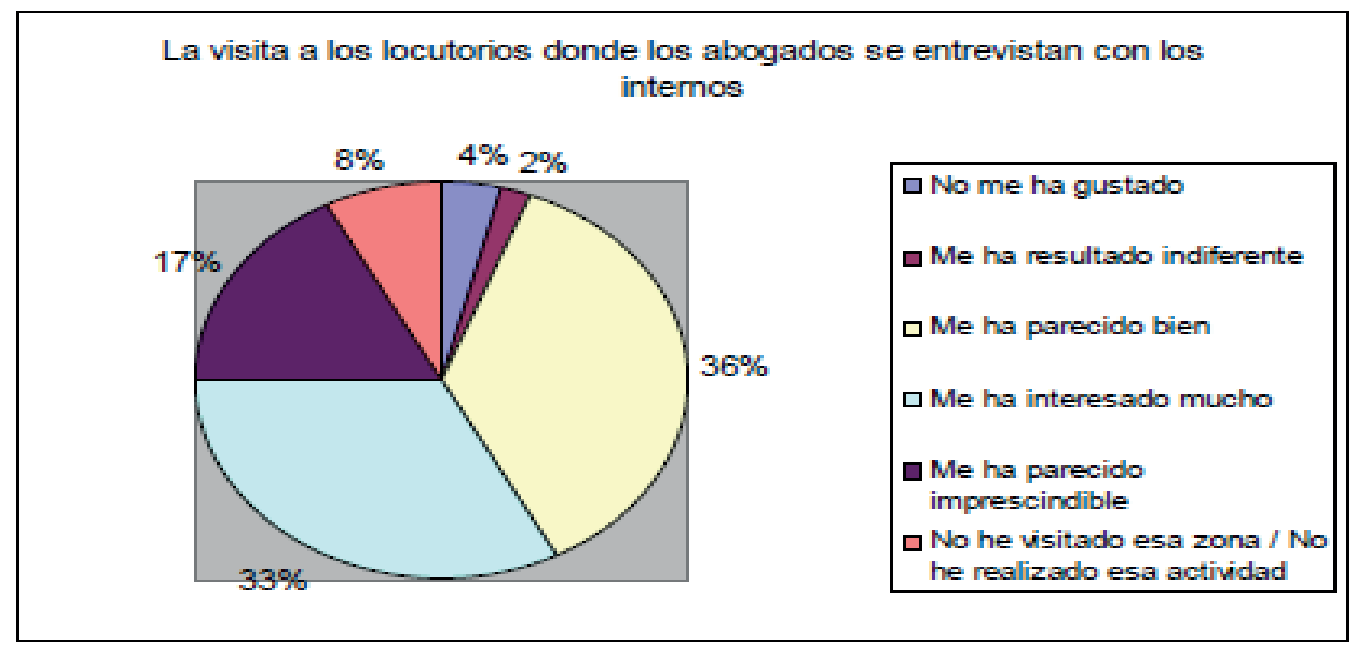

Figura 6. Visita a locutorios.

Fuente: elaboración propia.

Profundizando algo más en ello no carece de lógica el resultado si tenemos en cuenta, en primer lugar, que la valoración es muy positiva (a la mayoría les ha interesado mucho), y, en segundo lugar, que se trata de una parte del recorrido en la que simplemente se observa un pasillo dividido por una cristalera y con mesas y asientos a cada lado.

Por lo que respecta a la charla de bienvenida del funcionario, también la valoración es muy alta en la medida en que a la mayoría ha interesado mucho, si bien este punto no obtiene lo que podríamos denominar "la máxima calificación”. En el análisis de contenido de las preguntas abiertas pueden encontrarse ciertas razones para ello al no percibir al funcionario cercano a ellos y más bien nervioso, con ganas de terminar, o por pensar que lo más interesante es la charla con los propios presos y no con los funcionarios:

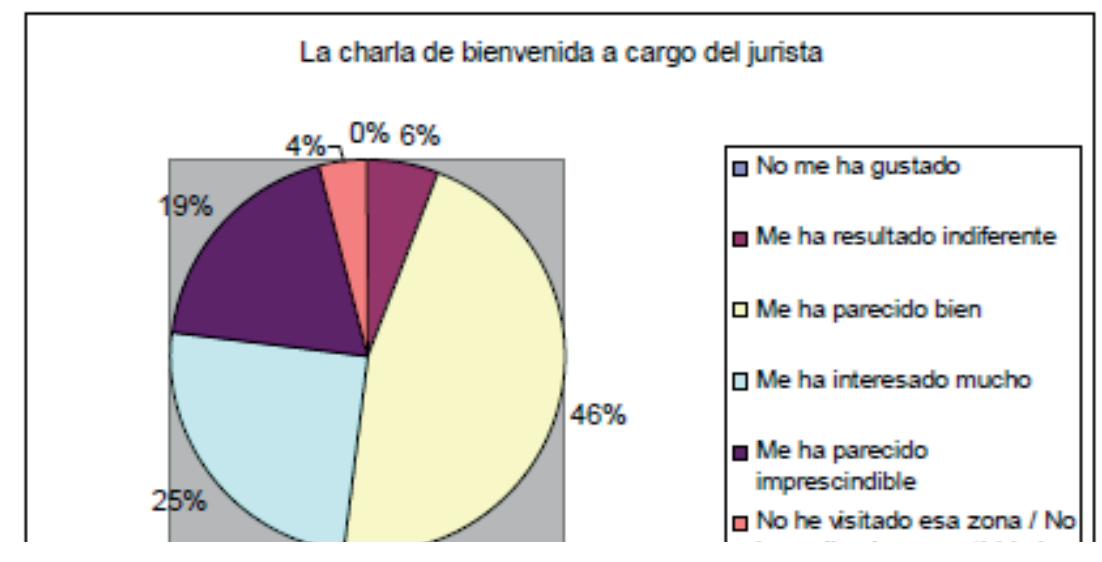


Por último, los alumnos son preguntados por su grado de acuerdo con una serie de afirmaciones acerca de la visita, cuyos resultados se muestran en la siguiente tabla:

Tabla 1. Medias (sobre 4 ptos.) en el acuerdo con diversas afirmaciones. Fuente: elaboración propia.

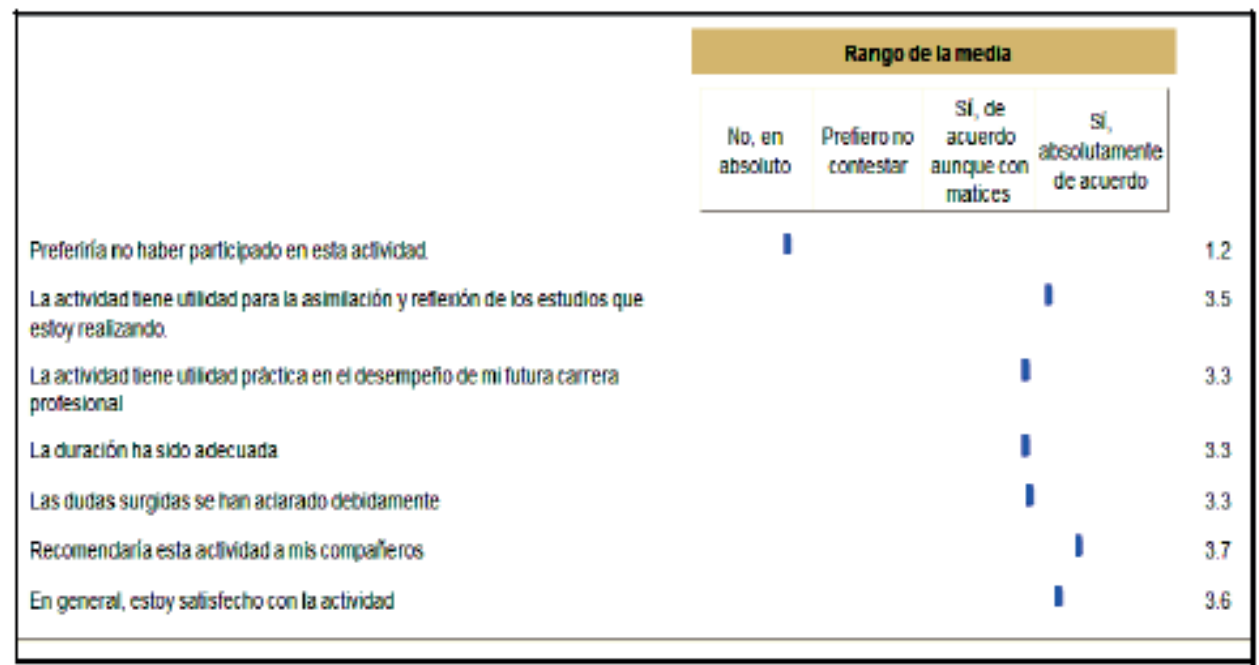

Es interesante ver que, en primer lugar, tan sólo el 6\% de los encuestados preferiría no haber participado (agrupando "Sí, absolutamente de acuerdo" y "Sí, de acuerdo, aunque con matices"), y cerca del 6\% prefiere no contestar. A pesar de ello, esto significa que el $88 \%$ está totalmente satisfecho de haber participado.

En cuanto a la utilidad de la actividad para la asimilación y reflexión del Derecho Penal, la media de acuerdo está en un 3,5 sobre 4 . Algo menos de acuerdo hay en torno a la utilidad práctica para la futura carrera profesional del estudiante, aunque la mayoría así lo considera. De la misma forma, tanto la duración, como la aclaración de dudas se considera adecuada con ciertos matices. Alguno de los encuestados se queja de no haber podido visitar más partes de la prisión, aunque normalmente comprenden las imposibilidades materiales de alargar la visita más de cuatro horas. Finalmente, la inmensa mayoría está satisfecho en general con la actividad y la recomendaría a sus compañeros.

De nuevo en el análisis de contenido de las preguntas abiertas, resultan llamativos varios comentarios que comparan la actividad con la visita a un zoo en el que se va a ver animales enjaulados. Los propios alumnos polemizan entre ellos al aludir a estos comentarios de sus compañeros y no estar de acuerdo con ellos. Se trasluce con ello

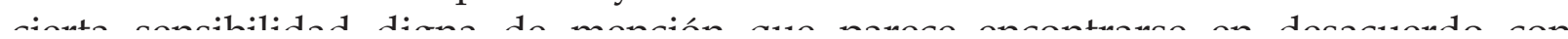




\section{CONCLUSIONES}

La valoración de los alumnos acerca de la visita que realizan al centro penitenciario es, por tanto, sumamente positiva. Teniendo en cuenta los problemas de tiempo que afrontan los profesores universitarios ante las mayores exigencias de dedicación a la docencia que el EEES trae consigo (Becerra Muñoz, 2011. Pág. 5), los datos extraídos de la encuesta muestran que el esfuerzo que cada año invierten los responsables de la asignatura tienen como resultado una actividad muy enriquecedora y satisfactoria para los estudiantes.

Dicho esto, para dar estos resultados por generalizables desde una perspectiva metodológica, es imprescindible seguir profundizando en la obtención de un mayor número de respuestas para poder así extrapolar estos resultados preliminares al resto de la población con mayor autoridad.

Por otra parte, la valoración de las diferentes actividades que se realizan durante la visita permite adoptar cierto orden de prelación para asegurar que se concede el debido tiempo e importancia a las actuaciones más productivas. Sin duda el contacto informal con los internos y funcionarios son los aspectos mejor valorados, de lo que se deduce la enorme utilidad de esta actividad para que los alumnos adquieran competencias tradicionalmente desatendidas en la docencia del Derecho Penal, como la adquisición de una conciencia crítica sobre la naturaleza represiva de esta rama del Derecho y sus instrumentos de control, así como de sus efectos secundarios no explícitos e impacto a nivel social y psicológico en los sujetos.

A este respecto, se constata en este estudio la sensibilidad de los alumnos en los temas tratados. Si bien en ocasiones se describe al estudiante universitario como un sujeto desinteresado, pasivo y difícil de motivar, los resultados de la encuesta nos muestran personas sensibles y comprensivas, incluso críticas en algunos casos con la degradación del ser humano que a su juicio supone la observación de los internos desde ciertos lugares, a través de cristales o rejas, o de sus pertenencias personales sin su presencia en las celdas.

Por el contrario, aquellas actividades con menos contacto humano, como la visita a ciertas zonas comunes que se suelen encontrar vacías en el momento de la visita, resultan menos interesantes para los alumnos, lo que, si bien no las hace inútiles, puede recomendar una menor dedicación a las mismas. Especialmente llamativa resulta la valoración de las visitas a los locutorios que, si bien se concibe habitualmente como importante, no despierta un interés tan alto como habríamos previsto. 
Un último aspecto hace coincidir a los alumnos encuestados con los profesores del Área de Derecho Penal, habitualmente contrarios a centrar las visitas en los módulos de internos menos conflictivos o de mujeres. En las primeras experiencias, las visitan incluyeron los módulos más conflictivos, consecuencia de lo cual los alumnos no tenían la sensación de realizar una visita "edulcorada" por los organizadores. A pesar de que la conflictividad de los centros penitenciarios no ha aumentado en los últimos años, sí lo ha hecho la prudencia de los sucesivos directivos del centro penitenciario, lo que ha acarreado que las visitas de los estudiantes se realicen sólo en módulos más "tranquilos". Aún así, los comentarios de los alumnos nos siguen animando a pedir al centro que las visitas incluyan módulos de reincidentes, extranjeros, preventivos, etc., en los que, en definitiva, se puede observar la parte más representativa de los centros penitenciarios españoles.

\section{REFERENCIAS}

Alonso Rimo, A. (2009). Aprendizaje cooperativo en Derecho Penal: Algunas Estrategias. En Miradas a la innovación: Experiencias de innovación en la docencia del Derecho. Valencia: Universidad de Valencia. Recuperado el 15 de julio de 2011, de http://www.uv.es/sfp.

Andrés ZambranA, L. \& Manzano Arrondo, V. (2004). ¿Hacia dónde camina la Universidad? Reflexiones acerca del EEES. Revista Interuniversitaria de Formación de profesorado, 18(3).

Bain, K., (2005). Lo que hacen los mejores profesores universitarios. Valencia: Publicaciones de la Universidad de Valencia.

Becerra Muñoz, J. (2011). Técnicas docentes para hacer posible la evaluación continua de alumnos con cargas familiares y laborales. Trabajo presentado al VIII Congreso Español de Criminología. SEIC.

Boldova Pasamar, M. A. \& Rueda Martín, Má Á. (2007). Un ejemplo de la aplicación de metodologías activas en las clases prácticas de la asignatura de Derecho penal, parte general. Trabajo presentado Innovación Docente, Tecnologías de la Información y la Comunicación e Investigación Educativa en la Universidad de Zaragoza. Caminando hacia Europa. Universidad de Zaragoza.

Bricall, J. (2000). Informe Universidad 2000. Barcelona. Recuperado el 30 de julio de 2010, de http:/ / www.oei.es/oeivirt/bricall.htm. 
Esteve, J. M. (2003). La tercera Revolución Educativa. La Educación en la Sociedad del Conocimiento. Barcelona: Paidós.

Fernández March, A. (2006). Metodologías activas para la formación de competencias. Educatio Siglo XXI, 24.

García-Borés Espí, J.M. (2003). El impacto carcelario. En Sistema penal y problemas sociales. Valencia: Tirant Lo Blanch.

García Magna, D.; Castillo Rodríguez, C.; Ríos Moyano, S., et. al. (2011). La interdisciplinariedad en la educación superior: Propuesta de una guía para el diseño de juegos de rol. TESI, 12(1). Recuperado el 22 de julio de 2011, de http://campus.usal.es/ revistas_trabajo/index.php/revistatesi/article/view/7894/ $\underline{7937}$.

García Magna, D. \& García España, E., (2011). La investigación criminológica como metodología docente innovadora. Trabajo presentado al VIII Congreso Español de Criminología. SEIC.

Gil Flores, J.; Álvarez Rojo, V.; García JiméneZ, E. \& Romero Rodríguez, S. (2004). La enseñanza universitaria, planificación y desarrollo de la docencia. Madrid: EOS.

González Rus, J.J. (2003). Reflexiones sobre el futuro de la enseñanza del derecho y sobre la enseñanza del derecho en el futuro. Revista electrónica de Ciencia penal y Criminología, 5(1). Recuperado el 21 de julio de 2011, de http://criminet.ugr.es/recpc/.

Gutiérrez Berlinchez, A.; De Prada Rodríguez, M. \& Cubillo López, I. J. (2010). Las visitas a los juzgados como actividad práctica para la docencia del Derecho procesal. Revista Electrónica de Investigación e Innovación Educativa, 2. Recuperado el 22 de julio de 2011, de http://www.eumed.net/rev/rejie/02/brl.htm. Consultado el.

Jiménez Martín, S. \& Moncholi Chaparro, M.A. (2009). El entrenamiento en técnicas creativas en el Espacio Europeo de Educación Superior. Trabajo presentado al I Congreso Internacional Latina de Comunicación Social Tenerife. Recuperado el 18 de julio de 2011, de http:// www.revistalatinacs.org/09/Sociedad/actas/12silvia.pdf.

Morin, E. (2001). La mente bien ordenada: repensar la reforma, reformar el pensamiento. Barcelona: Seix Barral.

Ortega y Gasset, J. (1930). Misión de la universidad. Madrid: Revista de Occidente. 
Ríos Corbacho, J. M. (2011). Innovación docente del Derecho Penal de la empresa a través de técnicas colaborativas y entornos virtuales de aprendizaje en el Espacio Europeo de Educación Superior. Revista Jurídica de Investigación e Innovación Educativa, 3. Recuperado el 18 de julio de 2011, de http://www.eumed.net/rev/rejie/.

Roldán Barbero, H. (2009): Introducción a la investigación criminológica. Comares.

Steiner, G. \& LadjalI, C. (2005). Elogio de la transmisión. Maestro y alumno. España: Ediciones Siruela.

Terradillos Basoco, J. M. (2008). Sobre el sentido de la enseñanza del Derecho Penal. Revista de Derecho Penal, 17.

Torrance, E.P. \& Myers, R.E. (1976). La enseñanza creativa. Madrid. Santillana

Varona Gómez, D. (2008). Ciudadanos y actitudes punitivas: Un estudio piloto de población universitaria española. Revista Española de Investigación Criminológica, 6. Recuperado el 2 de agosto de 2011, de http:/ / www.criminologia.net/pdf/reic/ano62008/a62008art1.pdf.

Zabalza Beraza, M. A. (2009). Competencias docentes del profesorado universitario. Trabajo presentado al VII Curso para Profesores Noveles de la Universidad de Málaga. 


\section{Deborah García Magna}

Profesora Colaboradora de Derecho Penal en la Facultad de Derecho de la Universidad de Málaga y Profesora-Investigadora en el Instituto Andaluz Interuniversitario de Criminología (Sección de Málaga). Desde el año 2001, ha impartido clases de Derecho Penal en la Licenciatura y el Grado en Derecho y en el Título de Experto en Criminalidad y Seguridad Pública, y de Derecho Penal y Derecho procesal penal en el Título de experto en Psicología Forense, además de participar como ponente en varios congresos de Criminología y un curso de verano sobre patrimonio arqueológico subacuático. Ha ejercido como abogada durante 9 años, especializándose en Derecho penal de menores, extranjería y violencia doméstica y de género. Sus áreas de interés como investigadora se centran en la política criminal de la seguridad ciudadana, las actitudes punitivas y su relación con los medios de comunicación y el populismo punitivo.

\section{José Becerra Muñoz}

Profesor ayudante de Derecho Penal en la Facultad de Derecho de la Universidad de Málaga y de Política Criminal en el Instituto Andaluz Interuniversitario de Criminología (Sección de Málaga). Es doctor europeus por la Universidad de Málaga y ha participado en diversos proyectos de investigación sobre política criminal, política legislativa penal y política penitenciaria, temas que, junto con la docencia en Criminología, han sido objeto de impartición de varias ponencias en congresos nacionales e internacionales. También ha participado en congresos y seminarios relacionados específicamente con el aprendizaje de técnicas docentes y la innovación docente, impartiendo un curso sobre "Nuevas metodologías docentes para la enseñanza de posgrado" en el Ilustre Colegio de Abogados de Málaga. Ha sido becario del Vicedecanato de Posgrado colaborando en la puesta en marcha y gestión de los másteres que imparte la Facultad de Derecho, coordinador del Boletín Criminológico y actualmente coordina el Observatorio de la Delincuencia (ODA) y el Aula de Investigación Novel de la Facultad de Derecho. 